\title{
Direct observation of spin-resolved full and empty electron states in ferromagnetic surfaces
}

Cite as: Rev. Sci. Instrum. 85, 073901 (2014); https://doi.org/10.1063/1.4885447

Submitted: 12 May 2014 . Accepted: 14 June 2014. Published Online: 02 July 2014

G. Berti, A. Calloni, A. Brambilla, G. Bussetti, L. Duò, and F. Ciccacci
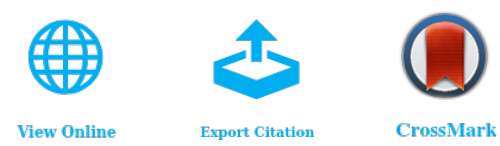

View Online

Export Citation

\section{ARTICLES YOU MAY BE INTERESTED IN}

The GaAs spin polarized electron source

Review of Scientific Instruments 51, 478 (1980); https://doi.org/10.1063/1.1136250

Electronic structure and bonding in metal porphyrins, metal=Fe, Co, Ni, Cu, Zn

The Journal of Chemical Physics 117, 205 (2002); https://doi.org/10.1063/1.1480872

Oxygen-induced immediate onset of the antiferromagnetic stacking in thin $\mathrm{Cr}$ films on Fe(001)

Applied Physics Letters 106, 162408 (2015); https://doi.org/10.1063/1.4918987

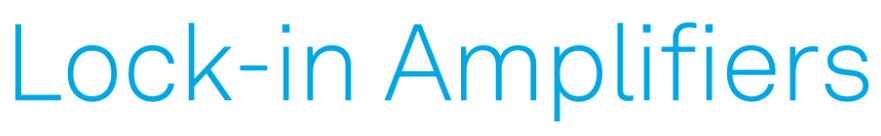

... and more, from DC to $600 \mathrm{MHz}$

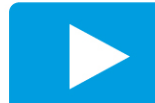

Watch 


\title{
Direct observation of spin-resolved full and empty electron states in ferromagnetic surfaces
}

\author{
G. Berti, a) A. Calloni, A. Brambilla, G. Bussetti, L. Duò, and F. Ciccacci \\ Dipartimento di Fisica, Politecnico di Milano, Piazza Leonardo da Vinci 32, I-20133, Milano, Italy
}

(Received 12 May 2014; accepted 14 June 2014; published online 2 July 2014)

\begin{abstract}
We present a versatile apparatus for the study of ferromagnetic surfaces, which combines spinpolarized photoemission and inverse photoemission spectroscopies. Samples can be grown by molecular beam epitaxy and analyzed in situ. Spin-resolved photoemission spectroscopy analysis is done with a hemispherical electron analyzer coupled to a $25 \mathrm{kV}$-Mott detector. Inverse photoemission spectroscopy experiments are performed with GaAs crystals as spin-polarized electron sources and a UV bandpass photon detector. As an example, measurements on the oxygen passivated $\mathrm{Fe}(100)-p(1 \times 1) \mathrm{O}$ surface are presented. () 2014 AIP Publishing LLC. [http://dx.doi.org/10.1063/1.4885447]
\end{abstract}

\section{INTRODUCTION}

Low dimensional magnetic systems including surfaces, interfaces, and thin films, are attracting much attention since several years because the lowered symmetry and coordination numbers, together with the presence of interface or surface states, offer a variety of opportunities for inducing new and exotic phenomena relevant in both basic physics and technological applications connected with digital data storage and spintronics. ${ }^{1}$ In particular, the reduced atomic coordination results in strong band narrowing with enhanced density of states at the Fermi level that, together with the electron localization, might enhance the spin magnetic moment. On the other hand, the symmetry breaking and the reduced crystal field splitting may partly remove the quenching of the orbital moment. ${ }^{2}$ One of the most common methods for investigating the electronic and magnetic properties of two-dimensional systems is based on spin-resolved electron spectroscopies. ${ }^{3}$ The reason is found in the short inelastic mean free path of electrons in solids, which leads to an escape depth (ED) of only a few atomic layers for typical electron energies between 10 and $1000 \mathrm{eV}$. Such a low ED allows a surface sensitive analysis, while information on the magnetic character is provided by the spin resolution. ${ }^{3}$ Among spin-resolved electron spectroscopies, a prominent role is covered by those based on photoemission. In fact, spin-polarized photoemission spectroscopy (SP-PES), giving direct access to spin-resolved occupied electronic states, is widely used for investigating magnetic thin films and surfaces. ${ }^{4,5}$ Moreover, the empty state region can be successfully probed by spin-polarized inverse photoemission spectroscopy (SP-IPES). ${ }^{6-8}$ The combined use of such spectroscopic techniques - without spin resolution has already shown to be an invaluable tool to derive, in particular, the energetics of electronic states at interfaces. ${ }^{9}$ The latter, in turn, play a prominent role in determining the mechanisms of charge transfer at the interfaces and charge transport in thin films. Relevant examples of applications currently of interest, just to mention a few, are metal/semiconductor junctions, ${ }^{10}$ organic/inorganic semiconductor junctions, ${ }^{11}$ organic films. ${ }^{12}$ The possibility of investigating the electronic

a)E-mail: giulia.berti@ polimi.it surface and interfaces of magnetic systems with both SP-PES and SP-IPES is therefore quite appealing and, moreover, a spin-polarized characterization of both occupied and empty energy states near the Fermi level in situ, on the very same sample and under the same experimental conditions, is still missing. ${ }^{13}$ Here we describe an experimental setup for the characterization of magnetic surfaces, equipped with both SPPES and SP-IPES facilities, with which we are able to investigate in situ both full and empty electron energy levels with spin resolution. After the description of the system, data on the well-known $\mathrm{Fe}(100)-p(1 \times 1) \mathrm{O}$ surface are shown as an application.

\section{APPARATUS}

The apparatus consists of an ultra-high vacuum system divided into three interconnected chambers: the first one is used for the preparation of the samples; the second one for the preparation of the photocathodes used as spin-polarized electron sources in SP-IPES experiments; finally, the third one is the main chamber in which the SP-PES and SP-IPES measurements are performed. The whole apparatus is sketched in Fig. 1.

The preparation chamber contains six molecular beam epitaxy cells with a quartz microbalance for the calibration of the deposition rates, a sputtering gun for ion bombardment, a low energy electron diffractometer (LEED) and a gas inlet for oxygen exposure. The second chamber is designed for the preparation of the spin-polarized electron sources: the electrons come from $\mathrm{GaAs}(100)$ photocathodes in negative electron affinity, ${ }^{14}$ whose surface is prepared with standard methods. ${ }^{15}$ The third chamber (see scheme in Fig. 1) is devoted to SP-PES and SP-IPES measurements. In both cases spectra are acquired in magnetic remanence (as usual in electron spectroscopies, see, e.g., Ref. 3), after having magnetized the sample by a current pulse sent to a coil surrounding it. Our system can produce magnetic fields up to some $0.1 \mathrm{~T}$, but much smaller values are generally required to remanently magnetize thin films. By moving the coils, an external magnetic field can be applied along two perpendicular directions, namely, along the manipulator axis ( $x$ axes in Fig. 1) and 


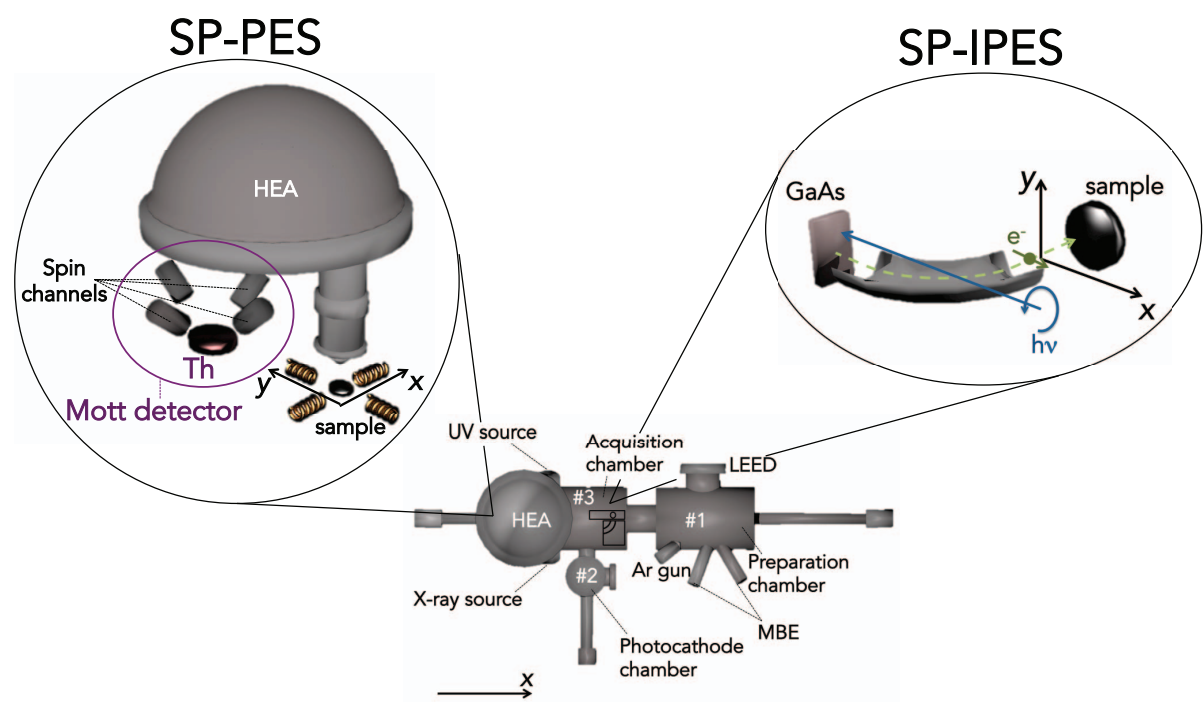

FIG. 1. Schematics of the experimental setup. The facilities for SP-PES and SP-IPES are highlighted; the magnetizing coils are shown just in the SP-PES inset for clarity. In the SP-IPES inset (right-hand side), the GaAs photocathode is excited with circularly polarized light, denoted with a blue arrow, and electrons are focused onto the sample by an electron optics which includes a spin rotator. The insets and the main picture are rotated with respect to each other as can be seen from $(x, y)$ axes directions. Note that, when going from the IPES position to the PES one, the sample is rotated by $90^{\circ}$ around the $x$ direction: being in our scheme the $y$ axis rigidly coupled to the sample, it undergoes a $90^{\circ}$ rotation passing from IPES to PES.

perpendicular to it ( $y$ axes in Fig. 1). Measurements can be taken at sample temperatures in the 30-400 $\mathrm{K}$ range, however all the data here presented have been taken at room temperature. SP-PES spectra are acquired by means of a 150 $\mathrm{mm}$ mean radius hemispherical electron analyzer (HEA); ${ }^{16}$ the photon sources are an unmonochromatized X-ray tube with $\mathrm{Mg}$ and $\mathrm{Al}$ anodes for the $\mathrm{X}$-ray photoemission spectroscopy (XPS) analysis and a He discharge lamp for ultraviolet photoemission spectroscopy (UPS). The system for SPIPES works in the isochromat mode: the impinging electron momentum and energy are controlled through a suitable electron optics system, while the outcoming photons in the UV range are detected by a bandpass detector. This system has been thoroughly discussed in Refs. 17-19 and will not be treated in detail here. We just refer to the fact that our SPIPES apparatus is only sensitive to the $x$ component of the sample magnetization, since after the spin rotator (see inset of Fig. 1 and Ref. 17) the electron beam is polarized along such direction.

Spin-polarized valence band and core levels spectra are acquired by means of a micro-Mott detector ${ }^{20}$ based on the "Rice University" design, ${ }^{21}$ mounted at the exit side of the HEA. The electrons at the image plane of the analyzer are detected with six standard channeltrons (three per side), while electrons in the central part of the exit window are fed through a $7 \mathrm{~mm}$ diameter aperture into a transfer acceleration lens, and focused onto a Th target biased at $25 \mathrm{kV}$. The electrons scatter from this target and, after decelerating to nearly ground potential, are detected by four channeltrons in the Mott detector: each pair of channeltrons serves to determine one spin direction. Instrumental asymmetries cancel each other out by acquiring spectra with two opposite directions of magnetization. A sketch of the position of the spin channels with respect to the analyzer is reported in Fig. 1. Channeltrons 1 and 2 measure the polarization perpendicular to the axis of the ma- nipulator, i.e., the $y$ axis of Fig. 1, while channeltrons 3 and 4 measure the parallel one ( $x$ direction in Fig. 1). Four spectra (one for each channeltron) are acquired in parallel for both signs of the magnetization.

The SP-PES system was calibrated by comparison with literature results on thin Fe/W(110) samples. ${ }^{22}$ For this purpose, Fe films have been evaporated in situ on an atomically clean W(110) surface prepared by heating the sample at about $1500 \mathrm{~K}$ in oxygen atmosphere and subsequently flash-heating it up to $2300 \mathrm{~K}$. UPS spectra were acquired using a pass energy of $7 \mathrm{eV}$. The sample was mounted in a way so to have one of the easy axes of magnetization parallel to the axis of the manipulator ( $x$ axis). When the Fe film thickness ranges from $20 \AA$ up to $60 \AA$, the easy axis of magnetization lies along the [110] direction, and the photoemitted electron spin polarization reaches a plateau at binding energies higher than $1 \mathrm{eV}$, while it changes sign at $0.4 \mathrm{eV}$ (see inset in Fig. 2). From the well known value of the polarization plateau $(80 \%$, see Refs. 22 and 23), we can get the value of the Shermann function (S) characterizing our Mott detector, finding $S=0.14$, which is well within the range of the specifications for this kind of devices. ${ }^{21}$

Spin-resolved spectra are then retrieved according to standard methods: ${ }^{4,5}$ normal emission valence band spectra from Fe/W(110) shown in Fig. 2 are in perfect agreement with literature results. ${ }^{22}$

As a further test we investigated the spin polarization dependence of core levels. Fig. 3 presents spin-resolved XPS spectra from a remanently magnetized Fe sample. Data have been acquired in the $2 p_{3 / 2}$ peak region at a pass energy of $20 \mathrm{eV}$ with $\mathrm{Mg} K_{\alpha}$ radiation (photon energy: $1253.6 \mathrm{eV}$, Fig. 3): the majority spin photoelectrons show a peak at a binding energy of $707.2 \pm 0.1 \mathrm{eV}$, while minority ones are located at $706.7 \pm 0.1 \mathrm{eV}$. Such a difference of about $0.5 \mathrm{eV}$ between the binding energies of majority and minority 


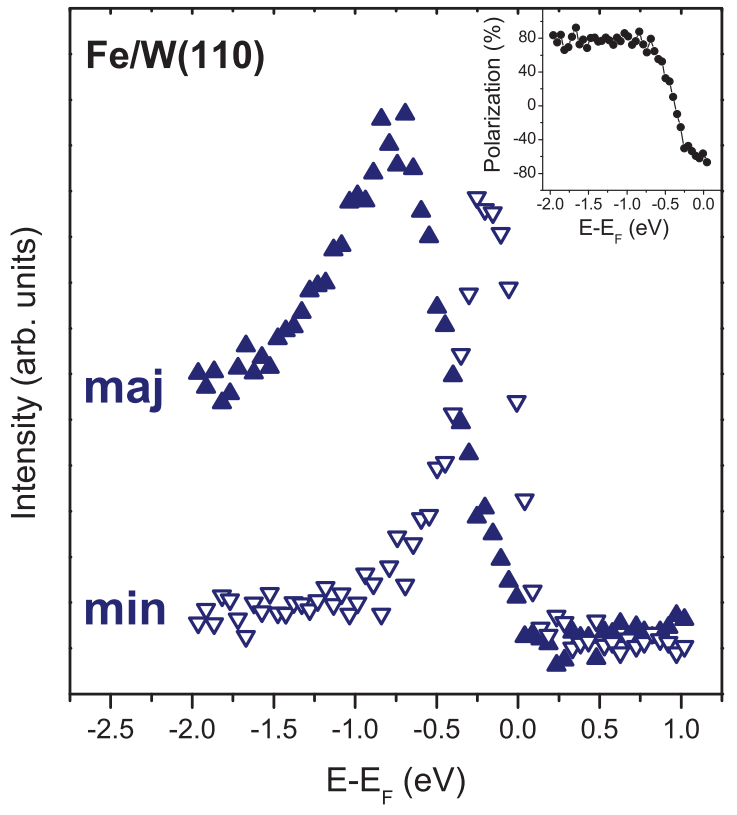

FIG. 2. Spin-resolved valence band spectra of $40 \AA \mathrm{Fe} / \mathrm{W}(110)$ at normal emission with $\mathrm{h} v=21.2 \mathrm{eV}$. Up triangles represent majority spins, down triangles minority ones. (Inset) Polarization (P) values. As fully described in Ref. 5, the polarization and the normalized plots are related by $\mathrm{P}=\left(I_{m a j}\right.$ $\left.-I_{\text {min }}\right) /\left(I_{\text {maj }}+I_{\text {min }}\right)$.

spin-splitted $2 p_{3 / 2}$ peaks are in full agreement with previous reports. $^{24}$

\section{SPIN-RESOLVED ELECTRON STATES IN $\mathrm{Fe}(100)-p(1 \times 1) 0$}

As an application of the system described above we present a study of the $\mathrm{Fe}(100)-p(1 \times 1) \mathrm{O}$ surface. Among low dimensional magnetic systems, in fact, such a surface rep-

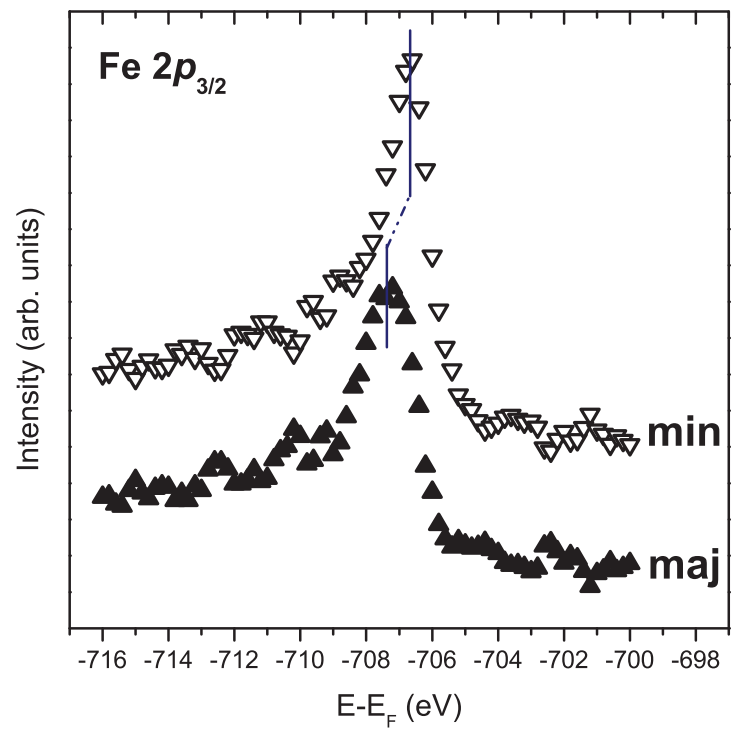

FIG. 3. Spin-polarized spectra taken in the core level $2 p_{3 / 2}$ region of a bulklike $\mathrm{Fe}(100)$ sample with $h v=1253.6 \mathrm{eV}$ normalized to the efficiency of the detector. Data are offset for clarity.

resents one of the most interesting workbenches, thanks to the high reproducibility in its preparation and to the oxygeninduced enhancement of spin-dependent effects. ${ }^{25-28}$ In the presence of a magnetic surface, it has been shown that an adsorbate can have a strong influence on the substrate magnetization, both in terms of local magnetic moments enhancement and of a possible induction of a magnetic moment in the adlayer. ${ }^{29,30}$ It is known that both possibilities occur when oxygen is chemisorbed on the $\mathrm{Fe}(100)$ surface keeping the $p(1 \times 1)$ symmetry, namely, when oxygen atoms occupy the fourfold hollow sites on top of the iron surface. ${ }^{31}$ The possibility of studying empty energy states with spin polarization

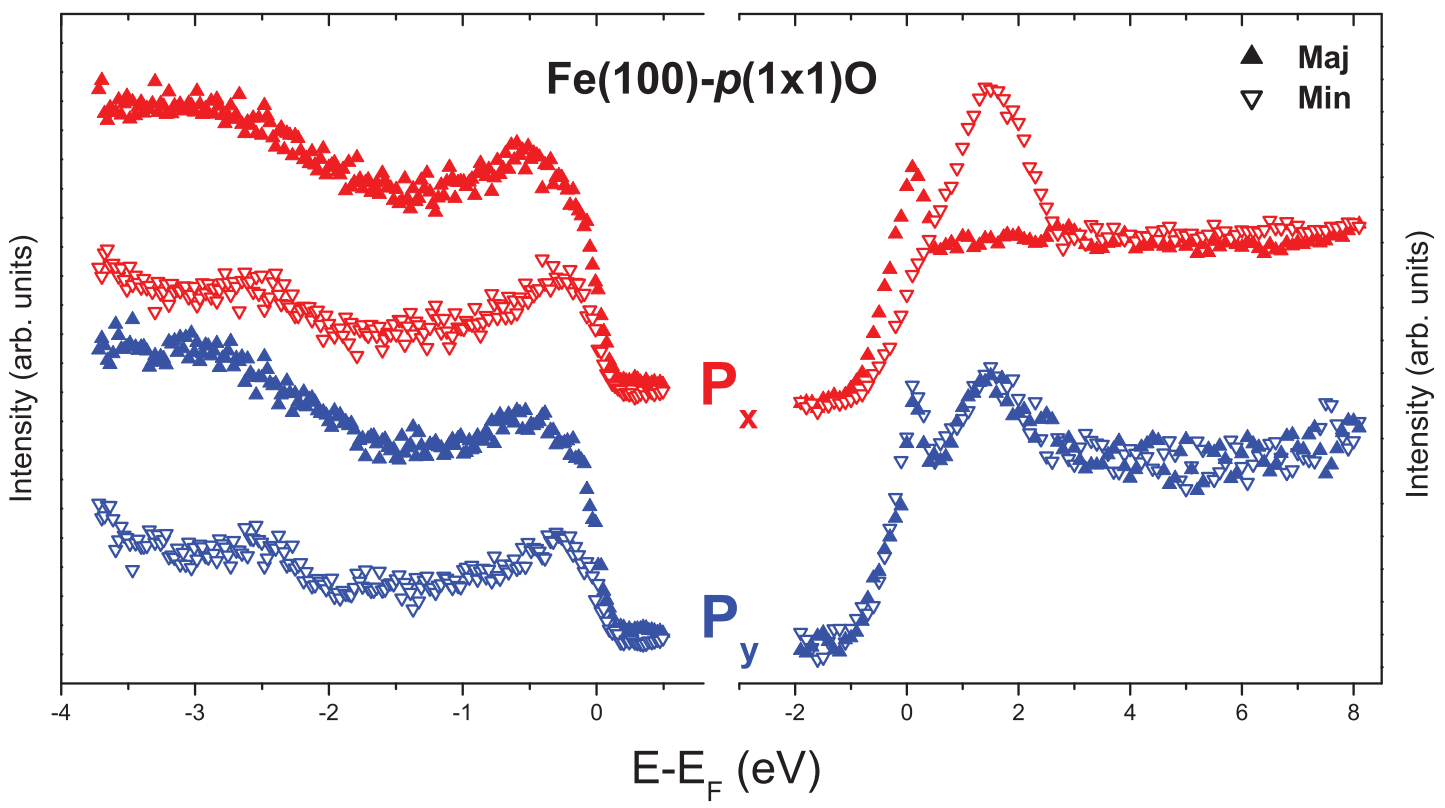

FIG. 4. Spin-polarized spectra taken just below and above the Fermi level on the $\mathrm{Fe}(100)-p(1 \times 1) \mathrm{O}$ sample. Up triangles represent majority spins, down triangles minority ones. For SP-IPES spectra a beam polarization of $27 \%$ was taken into account. SP-PES acquisitions were performed at a pass energy of $7 \mathrm{eV}$. The energy resolutions are 150 and $350 \mathrm{meV}$ (HWHM, half width at half maximum) for the SP-PES and SP-IPES measurements, respectively. Data acquisition time for each direction of polarization is about $30 \mathrm{~min}$ for SP-PES and twice as much for SP-IPES. 
has been exploited by our group by means of spin-polarized electron sources in inverse photoemission spectroscopy experiments. ${ }^{32,33}$ A great effort has also been made in the investigation of the magnetic properties of this surface with spin-polarized spectroscopies. ${ }^{34-36}$ Anyway, while several groups have studied the energy states below the Fermi level with the employment of synchrotron radiation sources, ${ }^{37,38}$ very little work has been done with ultraviolet discharge lamps, and only on clean, oxygen-free $\mathrm{Fe}(100)$ samples. ${ }^{39}$

The Fe- $p(1 \times 1) \mathrm{O}$ surface was obtained by exposing to $30 \mathrm{~L}\left(1 \mathrm{~L}=10^{-6}\right.$ Torr s) of pure grade molecular oxygen an atomically clean $\mathrm{Fe}(100)$ surface, which was successively flash-heated at $900 \mathrm{~K}$, as described in detail in Refs. 32, 35, and 36.

The experimental polarization projections $P_{y}$ (spin channels 1 and 2) and $P_{x}$ (spin channels 3 and 4) were obtained as illustrated in Ref. 5 for SP-PES. When the sample is magnetized along its easy axis in the $x$ direction (which, in this case, lies along one of the equivalent in-plane $\langle 100\rangle$ directions), spin channels 3 and 4 show an asymmetry in the counts. When, instead, the sample undergoes a magnetization parallel to the $y$ direction, channels 1 and 2 are the ones that report the asymmetry. The resulting spin-polarized spectra, normalized to the efficiency of the detector, show no qualitative differences, as seen in the left panel of Fig. 4.

SP-IPES data were also acquired on the same sample (both with magnetization along the two axes $x$ and $y$ ). Peaks relative to transitions towards majority and minority electrons are observed to be different for the two spin channels in the longitudinal $(x)$ magnetization, in agreement with previous results. ${ }^{32}$ Concerning the data obtained when magnetizing the sample along the $y$ direction, while the spectra still present the double peak structure related to exchange splitting between the two majority and minority empty states in $\mathrm{Fe},{ }^{32}$ no polarization dependence is seen (see right panel of Fig. 4), coherently with the fact that, as noted above, our facility allows us to be sensitive to the $x$ polarization of the surface in inverse photoemission experiments. ${ }^{40}$

\section{CONCLUSIONS}

We have described an experimental setup that allows for the investigation of full and empty electron energy levels in situ under the same experimental conditions with spin polarization, by means of spin-polarized photoemission spectroscopies. The inverse photoemission setup is sensitive to the surface polarization along one direction, while in photoemission from occupied states both the in-plane perpendicular surface directions are accessible. We have shown an application of the apparatus for the study of the $\mathrm{Fe}(100)-p(1 \times 1) \mathrm{O}$ surface.

\section{ACKNOWLEDGMENTS}

The authors thank R. Yivlialin and M. Finazzi for fruitful discussions and Y. Dedkov for advice on sample preparation. A.C. research is funded by Fondazione Cariplo (2012-09-04 SECARS project).
${ }^{1}$ A. Freeman and R. Wu, J. Magn. Magn. Mater. 100, 497 (1991).

${ }^{2}$ E. Stoner, Proc. R. Soc. London A 165, 372 (1938).

${ }^{3}$ R. Feder, in Polarized Electrons in Surface Physics, edited by R. Feder (World Scientific, Singapore, 1985).

${ }^{4}$ E. Kisker, Topics in Current Physics (Springer-Verlag, 1987), Vol. 42.

${ }^{5}$ P. Johnson, Rep. Prog. Phys. 60, 1217 (1997).

${ }^{6}$ V. Dose and M. Glöbl, in Polarized Electrons in Surface Physics (World Scientific, Singapore, 1985), p. 547.

${ }^{7}$ M. Donath, Surf. Sci. Rep. 20, 251 (1994).

${ }^{8}$ F. Ciccacci, Phys. Scripta T66, 190 (1996).

${ }^{9}$ D. Cahen and A. Kahn, Adv. Mater. 15, 271 (2003).

${ }^{10}$ Z. Zhang and J. Yates, Jr., Chem. Rev. 112, 5520 (2012).

${ }^{11}$ A. Brambilla, P. Sessi, L. Duò, M. Finazzi, J. Cabanillas-Gonzales, H.-J. Egelhaaf, G. Lanzani, and F. Ciccacci, Surf. Sci. 601, 4078 (2007).

${ }^{12}$ S. Krause, M. Casu, A. Schöll, and E. Umbach, New J. Phys. 10, 085001 (2008).

${ }^{13} \mathrm{~A}$ system similar to ours allowing for SP-PES and IPES has been recently described [S.-W. Yu, J. Tobin, and B. Chung, Rev. Sci. Instrum. 82, 093903 (2011)]. It was however focused on actinides and not on ferromagnetic films; moreover, no spin resolution was present for the empty part of the electronic states.

${ }^{14}$ D. Pierce, R. Celotta, G. Wang, W. Unertl, A. Galejs, C. Kuyatt, and S. Mielczarek, Rev. Sci. Instrum. 51, 478 (1980).

${ }^{15}$ F. Ciccacci and G. Chiaia, J. Vac. Sci. Technol. A 9, 2991 (1991).

${ }^{16}$ Phoibos 150 , SPECS ${ }^{\circledR}$ Surface Nano Analysis GmbH, Voltastrasse 5, 13355 Berlin, Germany, 2008.

${ }^{17}$ F. Ciccacci, E. Vescovo, G. Chiaia, S. De Rossi, and M. Tosca, Rev. Sci. Instrum. 63, 3333 (1992).

${ }^{18}$ M. Finazzi, A. Bastianon, G. Chiaia, and F. Ciccacci, Meas. Sci. Technol. 4, 234 (1993).

${ }^{19}$ G. Chiaia, S. De Rossi, L. Mazzolari, and F. Ciccacci, Phys. Rev. B 48, 11298 (1993).

${ }^{20}$ Spin Detector for Phoibos 150, SPECS ${ }^{\circledR}$ Surface Nano Analysis GmbH, Voltastrasse 5, 13355 Berlin, Germany, 2008.

${ }^{21}$ F. Dunning, Nucl. Instrum. Meth. Phys. Res. A 347, 152 (1994), and references therein.

${ }^{22}$ R. Kurzawa, K.-P. Kämper, W. Schmitt, and G. Güntherodt, Solid State Commun. 60, 777 (1986).

${ }^{23}$ M. Getzlaff, J. Bansmann, J. Braun, and G. Schönhense, Z. Phys. B 104, 11 (1997).

${ }^{24}$ D. Van Campen, R. Pouliot, and L. Klebanoff, Phys. Rev. B 48, 17533 (1993).

${ }^{25}$ F. Donati, P. Sessi, S. Achilli, A. Li Bassi, M. Passoni, C. Casari, C. Bottani, A. Brambilla, A. Picone, M. Finazzi, L. Duò, M. Trioni, and F. Ciccacci, Phys. Rev. B 79, 195430 (2009).

${ }^{26}$ A. Picone, G. Fratesi, A. Brambilla, P. Sessi, F. Donati, S. Achilli, L. Maini, M. Trioni, C. Casari, M. Passoni, A. Li Bassi, M. Finazzi, L. Duò, and F. Ciccacci, Phys. Rev. B 81, 115450 (2010).

${ }^{27}$ A. Picone, A. Brambilla, A. Calloni, L. Duò, M. Finazzi, and F. Ciccacci, Phys. Rev. B 83, 235402 (2011).

${ }^{28}$ M. Nyvlt, F. Bisio, J. Franta, C. Gao, H. Petek, and J. Kirschner, Phys. Rev. Lett. 95, 127201 (2005).

${ }^{29}$ A. Clarke, N. Brookes, P. Johnson, M. Weinert, B. Sinković, and N. Smith, Phys. Rev. B 41, 9659 (1990).

${ }^{30}$ S. Chubb and W. Pickett, Phys. Rev. Lett. 58, 1248 (1987).

${ }^{31}$ W. Feng, H. Meyerheim, K. Mohseni, O. Brovko, V. Stepanyuk, N. Jedrecy, R. Felici, and J. Kirschner, Phys. Rev. Lett. 110, 235503 (2013).

${ }^{32}$ R. Bertacco and F. Ciccacci, Phys. Rev. B 59, 4207 (1999).

${ }^{33}$ S. De Rossi, L. Duò, and F. Ciccacci, Europhys. Lett. 32, 687 (1995).

${ }^{34}$ B. Sinković, P. Johnson, N. Brookes, A. Clarke, and N. Smith, J. Appl. Phys. 70, 5918 (1991).

${ }^{35}$ R. Bertacco, M. Merano, and F. Ciccacci, Appl. Phys. Lett. 72, 2050 (1998).

${ }^{36}$ F. Bisio, R. Moroni, M. Canepa, L. Mattera, R. Bertacco, and F. Ciccacci, Phys. Rev. Lett. 83, 4868 (1999).

${ }^{37}$ E. Vescovo, O. Rader, and C. Carbone, Phys. Rev. B 47, 13051 (1993).

${ }^{38}$ L. Plucinski, Y. Zhao, C. Schneider, B. Sinković, and E. Vescovo, Phys. Rev. B 80, 184430 (2009).

${ }^{39}$ R. Bertacco, M. Marcon, G. Trezzi, L. Duò, and F. Ciccacci, Rev. Sci. Instrum. 73, 3867 (2002).

${ }^{40} \mathrm{An}$ apparatus sensitive to two perpendicular in-plane directions of the sample magnetization has been recently reported, see S. D. Stolwijk, H. Wortelen, A. B. Schmidt, and M. Donath, Rev. Sci. Instrum. 85, 013306 (2014). 